\title{
Biophotonics approach for the study of leukocyte activation
}

Chernova, Daria, Sokolovski, Sergei, Vorobev, Alexey Yu., Moskalensky, Alexander

Daria N. Chernova, Sergei G. Sokolovski, Alexey Yu. Vorobev, Alexander E. Moskalensky, "Biophotonics approach for the study of leukocyte activation," Proc. SPIE 11457, Saratov Fall Meeting 2019: Optical and Nano-Technologies for Biology and Medicine, 114570G (9 April 2020); doi: 10.1117/12.2560325

SPIE Event: Saratov Fall Meeting 2019: VII International Symposium on Optics and Biophotonics, 2019, Saratov, Russian Federation 


\title{
Biophotonics approach for the study of leukocyte activation
}

\author{
Daria N. Chernova ${ }^{a}$, Sergei G. Sokolovski ${ }^{\text {a,b }}$, Alexey Yu. Vorobeva,c and Alexander E. \\ Moskalensky*a,d \\ ${ }^{a}$ Novosibirsk State University, Pirogova str. 2, Novosibirsk, 630090 Russia \\ b Aston University, Aston Triangle, Birmingham, B4 7ET, UK \\ ${ }^{c}$ N.N. Vorozhtsov Novosibirsk Institute of Organic Chemistry SB RAS, 9 Lavrentiev Avenue, \\ Novosibirsk, 630090, Russia \\ ${ }^{\mathrm{d}}$ Voevodsky Institute of Chemical Kinetics and Combustion SB RAS, Institutskaya 3, Novosibirsk, \\ 630090 Russia
}

\begin{abstract}
Leukocytes are the main cells of immune system, but also contribute to other systems and participate in pathogenesis of different diseases. In particular, leukocytes are involved in the progression of diabetic retinopathy due to their hyperactivation in diabetes. However, a connection between diabetes and the dysfunction of leukocytes is poorly understood. For a more complete picture, studies of the leukocytes activation under the influence of various substances are necessary. Arachidonic acid (AA) and its metabolites are the strongest activating factors of leukocytes. However, the studies involving AA are complicated because it is water-insoluble. Here we describe the method to study activation using photolabile analogs of AA.
\end{abstract}

Keywords:, arachidonic acid, photolabile analogs, Jurkat cell, intracellular calcium, caged arachidonic acid, single cell optical control, biophotonics, dysfunction of leukocytes.

\section{INTRODUCTION}

Currently, diabetes is one of the most common chronic diseases. The prevalence of diabetes in human populations is from 1 to $8.6 \%$ on average. According to the International Diabetes Federation (IDF) on January 2016, about 415 million people aged 20 to 79 years in the world suffer from diabetes. Death from the disorder and its complications occurs every seven seconds, with 50\% of these deaths (totaling 4 million per year) occurring before the age of 60 .

Diabetic retinopathy is one of the most severe complications of diabetes mellitus. It is the most common cause of partial vision loss and blindness in people over 20 years of age ${ }^{1}$. The cause of retinopathy is an impaired blood flow in the retina. Currently, it is accepted that main players in this process are leukocytes, which in diabetes are hyperactivated, easily become "sticky" and block blood vessels 2 . It primarily leads to the blockage of narrow eye capillaries, which in turn causes depletion of the retinal area with oxygen. However, a connection between diabetes and the dysfunction of leukocytes is poorly understood. For a more complete picture of the pathogenesis of diabetic retinopathy, studies of the leukocytes activation under the influence of various substances are necessary.

Arachidonic acid (AA) and its metabolites are the strongest activating factors of leukocytes. AA is a polyunsaturated fatty acid that is a precursor to eicosanoids, leukotrienes and trombaxanes. These products are extremely important in many cellular processes and essential for many organs and systems to function. However, many effects of AA was found to be 
independent of these products: it can directly modulate phagocytosis, cytokine production, expression of surface molecules, white blood cell migration, and antigen presentation ${ }^{3}$.One of the known mechanisms of action of AA is to stimulate the generation of superoxide, which acts as a microbicidal agent in phagocytes ${ }^{4}$. This fact can also be associated with the cytotoxicity of $\mathrm{AA}^{5}$.

In this paper, we study the activation of leukocytes triggered by AA, in particular, the dynamics of early calcium response. According to the literature, maximum concentration peak is reached in the first 30 seconds in human neutrophils, while a sharp increase in intracellular calcium occurs within 3 minutes $^{6}$. Immunofluorescence studies of B lymphocytes showed that the response time to the addition of AA can take up to 3 minutes, but the jump in intracellular calcium concentration takes one or two seconds?

Usually, the study of the effects of AA on cells using microscopy is associated with the following problems. First, AA is not water-soluble acid and it makes oil-in-water emulsion. Therefore, simple addition of AA to the suspension of cells is not enough to reach all cells. But any mixing takes time during which the early reaction occurs. As a result, the concentration of AA cannot be definitely determined and may vary from cell to cell. Second, addition of any substance makes the cells being monitored to shift or even completely float away from the observation area. Third, the time where the compound started acting on cells is undefined because of uncontrolled diffusion step. To overcome all these problems, we prepared photolabile ("caged") analogs of AA, which do not activate cells but generate AA under UV illumination. The use of these analogs allows us to add the substance in advance, giving it sufficient time to dissolve. Then it allows to trigger the reaction by flash of UV light, and thus gives us the opportunity to study its dynamics in more detail.

\section{MATERIALS AND METHODS}

\subsection{Compounds and chemical synthesis}

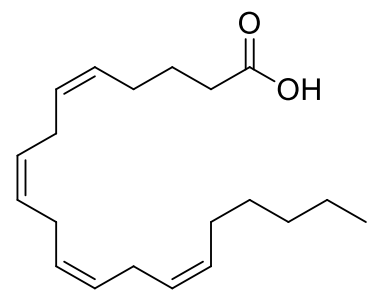

Arachidonic acid

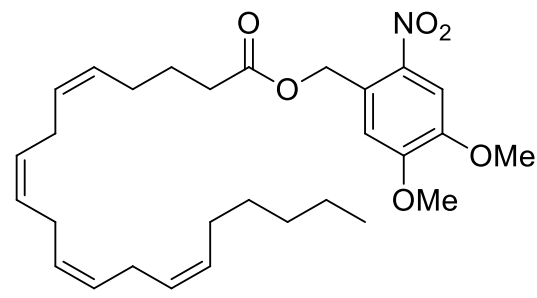

Analog 1<smiles>CCCCC/C=C\C/C=C\C/C=C\C/C=C\CCCC(=O)OC(C)c1cc(OC)c(OC)cc1[N+](=O)[O-]</smiles>

Analog 2

Figures 1: (a) Arachidonic acid (AA) and its two photolabile analogs.

Two modified versions of arachidonic acid were synthesized by conjugation with photoremovable protective groups. Their structural formulas are shown in Figure 1.

For the synthesis of substances, either (4,5-dimethoxy-2-nitrophenyl)methanol for analog 1 or 1-(4,5-dimethoxy-2nitrophenyl)ethan-1-ol for analog 2 were dissolved in $\mathrm{CH} 2 \mathrm{Cl} 2$, and then $\mathrm{NEt} 3$ and acetyl chloride were added to the resulting mixture. After that, reaction mixture was thoroughly mixed for half an hour, then transferred to a separatory funnel and washed. Organic layer was separated, dried and evaporated in vacuo. Solid residue was purified by column chromatography $(\mathrm{SiO} 2, \mathrm{CH} 2 \mathrm{Cl} 2)$ and thus both analogues were synthesized. ${ }^{8}$.

Both compounds provide free arachidonic acid after photolysis. The UV-Vis absorption spectrum of the first analog was demonstrated at the previous article ${ }^{9}$. The spectrum of second analog during decomposition is showed on Figure2. 


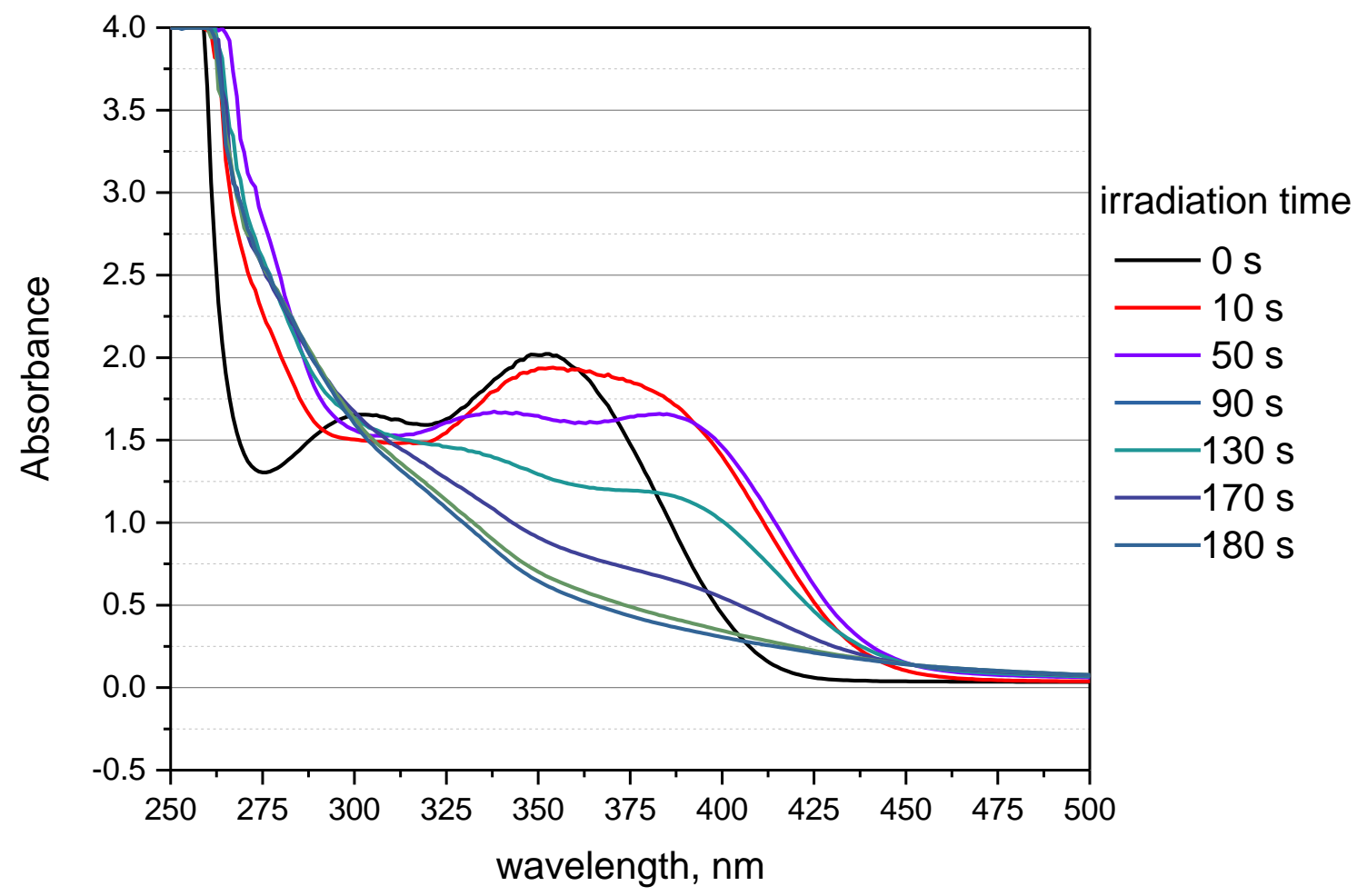

Figures 2: The absorption spectrum of analog 2 before and during photolysis. Under the action of UV, an initial spectrum (black line) approach the AA spectrum (blue line).

\subsection{Sample preparation.}

The cell line used in this study was of human origin. Jurkat cells were kindly provided to us by colleagues from Novosibirsk scientific center. Cells were grown in IMDM basic medium supplemented with L-Glutamine, 25mM HEPES, $10 \%$ fetal bovine serum and $10 \mathrm{U} / \mathrm{ml}$ gentamicin in an incubator at a temperature of $37^{\circ} \mathrm{C}$ and a concentration of $\mathrm{CO}_{2}$ of $5 \%$. The cell population was maintained between $1 \times 10^{5}$ and $1 \times 10^{6} \mathrm{cells} / \mathrm{ml}$ (logarithmic phase of cell growth). Fluo 4 calcium indicator was diluted in IMDM with gentamicin and added to cell (final concentration $2 \mu \mathrm{M}$ ). The sample was then incubated for 1 hour in the dark in the incubator. Cell-permeant acetoxymethyl ester of Fluo4 was used (Invitrogen ${ }^{\mathrm{TM}}$, cat. No F14217), which undergoes de-esterification inside cells and then shows an increase in fluorescence upon $\mathrm{Ca}^{2+}$ binding. After the incubation, cells were again resuspended in 9-fold volume of IMDM, and then allowed to rest for another $30 \mathrm{~min}$ to decrease non-specific binding and complete the de-esterification. All measurements were carried in plastic Petri dish for microscopy.

\subsection{Optical system}

To record changes in intracellular calcium we used the inverted microscope (Carl Zeiss AxioVert.A1). For a more detailed observation of the reaction, the microscope was equipped with a highly sensitive camera AxioCam 503 mono.

In order to provoke the detachment of the protective "caging" group by irradiation with ultraviolet radiation, we used Nichia NVSU233A LED with $365 \mathrm{~nm}$ central wavelength and $1030 \mathrm{~mW}$ radiant flux. LED was fixed over a petri dish in which the sample was placed (Figure 1). Arduino Due board was employed for the control of irradiation length. 


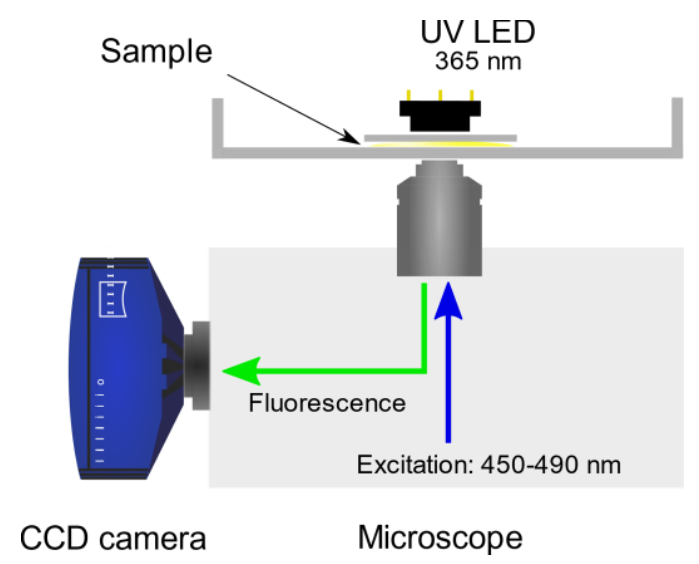

Figure 1. A. Schematic layout of experimental setup. Inverted microscope (Carl Zeiss AxioVert.A1) is used to monitor intracellular calcium concentration in leukocytes. The activation was achieved using arachidonic acid (AA) and two its photolabile analogs which provides AA upon UV irradiation.

\section{RESULTS}

\subsection{Activation of leukocyte by AA}

To start the development of our method, we first conducted experiments with conventional (unmodified) arachidonic acid to develop a research protocol and optimize all parameters. It is known that the addition of arachidonic acid causes an increase in the concentration of intracellular calcium. In this case, the average fluorescence signal of leukocytes should increase.

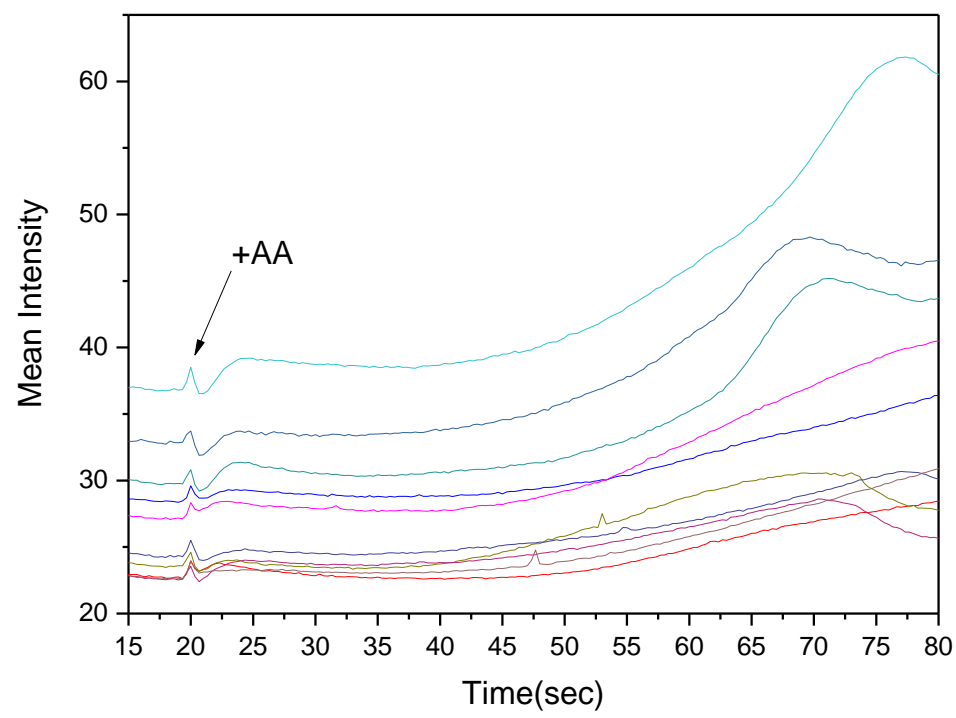

Figure 3: Changes in intracellular calcium concentration (Fluo-4 fluorescence intensity) under the influence of AA. Each graph corresponds to one cell. 
We added $10 \mu \mathrm{l}$ of arachidonic acid diluted in DMSO (final concentration $0.12 \mathrm{mM}$ ) to $400 \mu \mathrm{l}$ of a sample containing approximately $2.5 \cdot 10^{6}$ cells. We observed a twofold increase in the fluorescence intensity. Despite the fact that the concentration of intracellular calcium in different cells does not coincide, the increase in intensity occurs simultaneously with a comparable rate (Figure 3). For some cells (the largest in size, and in addition, having a high concentration of intracellular calcium), we also observed a slow decline one minute after the addition of acid. It should also be said that there were cells in the population that did not respond to the addition of AA. They were probably dead (damaged) during sample preparation. In order to exclude the influence of solvent, we added pure DMSO to the cells in the same proportions (10 $\mu \mathrm{l}$ to $400 \mu \mathrm{l})$, and no reaction was observed.

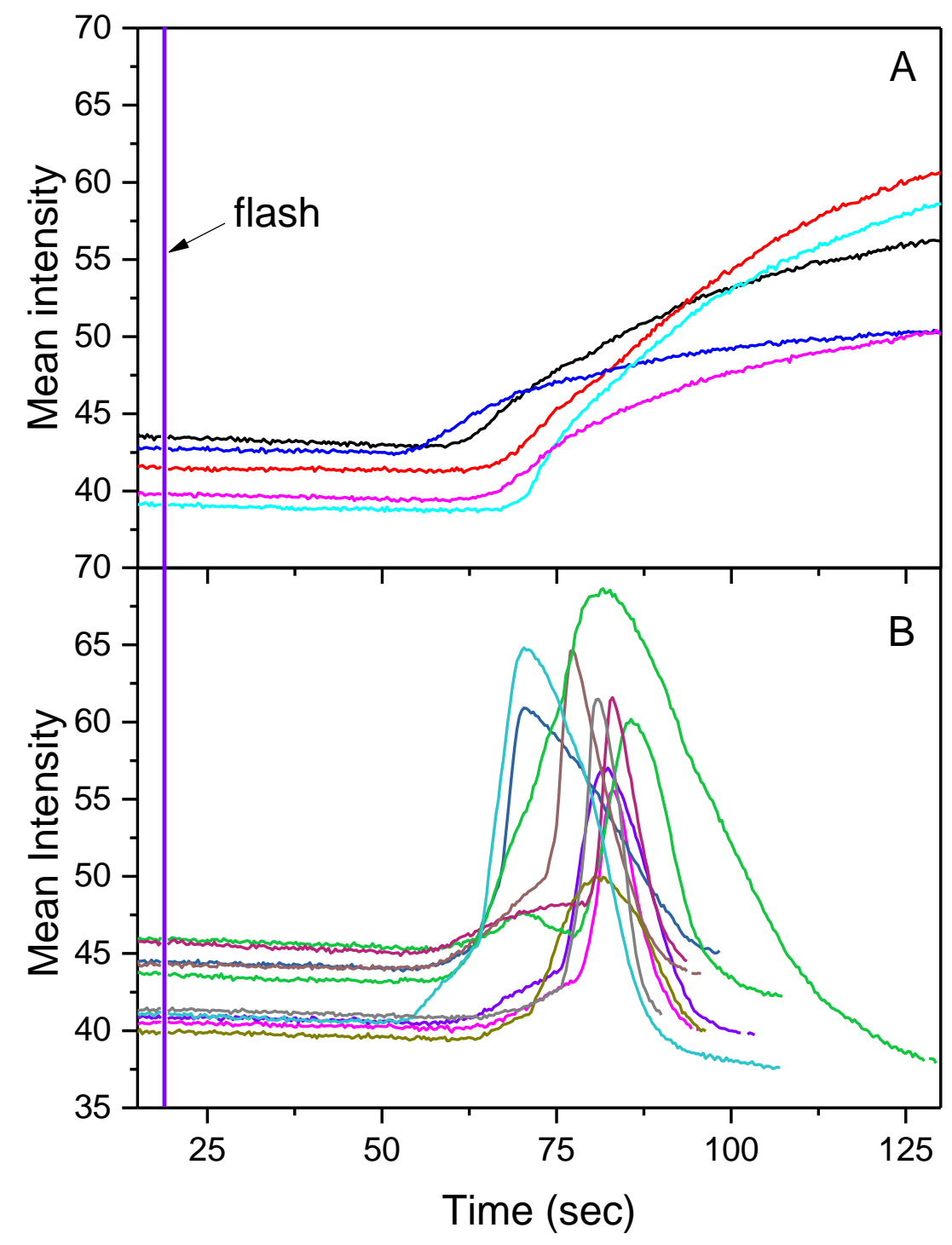

Figure 4: Changes in intracellular calcium concentration (cell fluorescence intensity) under the influence of analog 1. (A) Population 1: Intensity increase (B) Population 2: Peak Intensity, rise and fall. 
After the necessary proportions were established, an experiment was conducted with analogue 1 . It was added to $400 \mu$ of the sample in a volume of $10 \mu \mathrm{l}$ (final concentration $6 \mathrm{mM}$ ). Then 10 minutes were allotted so that the analog could completely dissolve. After the flash, we observed three types of cell responses.

The first population experienced a sharp increase in intensity 30 seconds after the flash (Figure 4A). At the time when recording was stopped (after 3 minutes), the intensity continued to increase. Over this period, the average population intensity (of cells with this type of reaction) increased by $60 \%$.

In the second population, the following course of the reaction was observed: the intensity increased sharply (the speed of the reaction of increase in intensity in this population is seven times higher than the speed of the first population) and also sharply fell (Fig. 4B). Some cells after a bright spark became invisible. We suggest that the increase in intracellular calcium concentration in these cells was so intense that the cells were blown out.

There are also a small number of cells that do not respond to the flash; their intensity has not changed in any way during the measured period.

\section{DISCUSSION}

This described activation method is promising for our study. However, several problems still need to be solved. Firstly, experimental protocols (labeling and incubation) should be optimized to maximally preserve the ability of cells to react to the environment. Secondly, the observed calcium response is directly caused by leukotrienes, which in turn are the products of AA metabolism in the cytoplasm. However, after the formation leukotrienes should go outside cell to induce activation (so called autocrine and paracrine pathways). All the parameters of this complex process should be accounted for to achieve a reliable activation response. Further studies are needed fo this purpose. The development of method for leukocytes can serve as a basis for research of other cells and processes.

\section{DISCLOSURES}

No conflicts of interest, financial or otherwise, are declared by the authors.

\section{ACKNOWLEDGEMENTS}

This work was supported by the Grant of President of Russian Federation for state support of young scientists (Grant agreement 075-15-2019-1034 (MK-1715.2018.2)).

\section{REFERENCES}

[1] "IDF Diabetes Atlas 9th edition 2019," <https://diabetesatlas.org/en/> (18 November 2019).

[2] Fu, X., Gens, J.S., Glazier, J.A., Burns, S.A., and Gast, T.J., "Progression of Diabetic Capillary Occlusion: A Model," PLOS Computational Biology 12(6), e1004932 (2016).

[3] Pompéia, C., Lopes, L.R., Miyasaka, C.K., Procópio, J., Sannomiya, P., and Curi, R., "Effect of fatty acids on leukocyte function," Brazilian Journal of Medical and Biological Research 33(11), 1255-1268 (2000).

[4] Suzuki, Y.J., Forman, H.J., and Sevanian, A., "Oxidants as Stimulators of Signal Transduction," Free Radical Biology and Medicine 22(1-2), 269-285 (1997). 
[5] Pompeia, C., Freitas, J.J.S., Kim, J.S., Zyngier, S.B., and Curi, R., “Arachidonic acid cytotoxicity in leukocytes: implications of oxidative stress and eicosanoid synthesis," Biology of the Cell 94(4-5), 251-265 (2002).

[6] Naccache, P.H., McColl, S.R., Caon, A.C., and Borgeat, P., "Arachidonic acid-induced mobilization of calcium in human neutrophils: evidence for a multicomponent mechanism of action," British Journal of Pharmacology 97(2), 461-468 (1989).

[7] Zhu, P., Liu, X., Labelle, E.F., and Freedman, B.D., "Mechanisms of Hypotonicity-Induced Calcium Signaling and Integrin Activation by Arachidonic Acid-Derived Inflammatory Mediators in B Cells," The Journal of Immunology 175(8), 4981-4989 (2005).

[8] Lage Robles, J., and Bochet, C.G., "Photochemical Release of Aldehydes from $\alpha$-Acetoxy Nitroveratryl Ethers," Organic Letters 7(16), 3545-3547 (2005).

[9] Vorob'ev, A.Yu., Dranova, T.Yu., and Moskalensky, A.E., "Photolysis of dimethoxynitrobenzyl-'caged' acids yields fluorescent products," Scientific Reports 9(1), 13421 (2019). 